\title{
Influência da Irradiação Solar na Análise de Viabilidade Econômica de Sistemas Fotovoltaicos
}

\author{
Fabrício Higo Monturil de Morais ${ }^{1}$ (D), Osvaldo Augusto Vasconcelos de Oliveira \\ Lopes da Silva $^{1}$ (D), Albemerc Moura de Moraes ${ }^{2}$ (D), Fábio Rocha Barbosa ${ }^{3}$ \\ ${ }^{1}$ Instituto Federal de Educação, Ciência e Tecnologia do Piaui, Campus Teresina-Central, \\ Teresina, PI, Brasil. \\ ${ }^{2}$ Universidade Federal do Piauí, Campus Senador Helvídio Nunes, Picos, PI, Brasil. \\ ${ }^{3}$ Universidade Federal do Piauí, Campus Ministro Petrônio Portela, Teresina, PI, Brasil.
}

Recebido em: 6 de Julho de 2021 - Aceito em: 15 de Setembro de 2021

\begin{abstract}
Resumo
A instalação de sistemas fotovoltaicos mostra-se uma alternativa promissora para a geração sustentável de energia elétrica. A realização de estudos de viabilidade de instalações fotovoltaicas é de extrema importância para se verificar a disponibilidade do recurso solar no local de instalação. Esta pesquisa tem como objetivo comparar os resultados da análise econômica de um sistema fotovoltaico com diferentes estimativas de geração de energia elétrica, utilizando diferentes bases de dados de irradiação solar. Tendo como objeto de estudo o sistema fotovoltaico do campus Floriano do IFPI, foram obtidos dados de irradiação solar a partir de diferentes bancos e softwares dedicados, e estimada a produção de energia elétrica. Esta foi comparada com os dados reais de geração, obtidos através do software de monitoramento dos inversores do sistema, e submetida a testes estatísticos. Para cada amostra foi realizado um estudo econômico, utilizando os conceitos da engenharia econômica. Os resultados mostram que a escolha da metodologia de cálculo das estimativas de geração pode influenciar diretamente nos resultados das análises de viabilidade, o que pode levar a escolhas erradas. Dependendo do tamanho do erro, problemas podem surgir durante a utilização do sistema a curto, médio e longo prazo.
\end{abstract}

Palavras-chave: sistemas fotovoltaicos, irradiação solar, avaliação econômica.

\section{Influence of Solar Irradiation in the Analysis of the Economic Viability of Photovoltaic Systems}

\begin{abstract}
The installation of photovoltaic systems is a promising alternative for the sustainable generation of electricity. Carrying out feasibility studies for photovoltaic installations is extremely important to verify the availability of the solar resource at the installation site. This research aims to compare the results of the economic analysis of a photovoltaic system with different estimates of electricity generation, using different databases of solar irradiation. Having as object of study the photovoltaic system of the Floriano campus of the IFPI, solar irradiation data were obtained from different banks and dedicated software, and estimated the production of electricity. This was compared with the actual generation data, obtained through the monitoring software of the system's inverters, and submitted to statistical tests. For each sample, an economic study was carried out, using the concepts of economic engineering. The results show that the choice of methodology for calculating generation estimates can directly influence the results of the feasibility analyses, which can lead to wrong choices. Depending on the size of the error, problems can arise when using the system in the short, medium and long term.
\end{abstract}

Keywords: photovoltaic systems, solar irradiation, economic evaluation.

Autor de correspondência: Fabrício Higo Monturil de Morais, fabricio@ifpi.edu.br. 


\section{Introdução}

Com o aumento da população os seres humanos passam a necessitar de mais energia para criar um ambiente de vida melhor (Sampaio e González, 2017). Por conta disso, o consumo global de energia vem aumentando a cada ano de forma exponencial. No entanto, a taxa de restabelecimento de recursos, como carvão, petróleo e gás natural é muito lenta em comparação à taxa de consumo. Assim, se torna muito importante a busca de tecnologias que empregam recursos energéticos sustentáveis (Bashir et al., 2018).

A energia solar fotovoltaica vem sendo a melhor opção para o futuro por ser a fonte de energia mais abundante na natureza, proporcionando eficiências de produção sólidas e crescentes (Kannan e Vakeesan, 2016). Além disso, tem poucos impactos nocivos ao meio ambiente, relacionados apenas com a etapa de produção das células fotovoltaicas (Silva et al., 2018). Dessa maneira, vem sendo utilizada em diversas aplicações, como, por exemplo, fonte de energia para bombeamento de água, alimentação de instalações remotas, residências urbanas e telecomunicações (Parida et al., 2011).

No Brasil, inicialmente, seu uso englobava, em sua maioria, sistemas isolados em pequena escala em áreas sem conexão com o Sistema Interligado Nacional (SIN) (Holdermann et al., 2014). No entanto, a quantidade de instalação de sistemas de geração fotovoltaicos conectados à rede elétrica no Brasil veio a crescer nos últimos anos. Isso se deu principalmente após a publicação da Resolução Normativa $n^{\circ}$ 482/2012 da Agência Nacional de Energia Elétrica (ANEEL), que padronizou a inclusão da energia fotovoltaica na matriz energética brasileira, através das modalidades de micro e minigeração distribuídas (ANEEL, 2012). Outro marco importante para a energia fotovoltaica no Brasil ocorreu em 2015, com a publicação da $\mathrm{RN} \mathrm{n}{ }^{\circ}$ 687/2015 da ANEEL, que alterou a resolução anterior e estabeleceu condições e facilidades para a utilização da geração distribuída, para os anos seguintes. Com esta atualização, a potência permitida para a microgeração, que era de até $100 \mathrm{~kW}$, passou a ser $75 \mathrm{~kW}$, e para a minigeração, que era permitida de $100 \mathrm{~kW}$ a $1 \mathrm{MW}$, passa a valer de $75 \mathrm{~kW}$ a $5 \mathrm{MW}$ (ANEEL, 2015).

Assim, como consequência destas ações regulatórias combinadas com os avanços na tecnologia fotovoltaica, verificou-se um aumento significativo da geração distribuída no Brasil, principalmente na área de sistemas conectados à rede elétrica (Vale et al., 2017). De acordo com o Plano Decenal de Energia (PDE) 2016-2026, publicado pela Empresa Pesquisa Energética (EPE) do Ministério de Minas e Energia (MME), estimam-se cerca de 770 mil adotantes de sistemas fotovoltaicos sob o regime da $\mathrm{RN}$ $\mathrm{n}^{\circ} 482 / 2012$, totalizando $3,3 \mathrm{GW}$, suficiente para atender $0,6 \%$ do consumo total nacional, em 2026 (EPE, 2017b). Ademais, em 2016, a micro e a mini geração distribuída atingiram a marca dos $359,15 \mathrm{GWh}$ gerados, com uma potência instalada de $246,1 \mathrm{MW}$, com destaque para a fonte solar fotovoltaica, com 165,87 GWh e 174,5 MW de geração e potência instalada respectivamente (EPE, 2017a).

A instalação de sistemas fotovoltaicos pode ser uma alternativa viável economicamente e um diferencial nas ações de economia de energia elétrica tendo em vista o grande potencial para a utilização desta fonte. No entanto, é preciso entender que a difusão da energia solar traz benefícios não somente energéticos e ambientais, mas também sociais e econômicos (Sá et al., 2018). A combinação de tarifas residenciais elevadas com alta disponibilidade de radiação solar, são fortes indicativos de que a eletricidade fotovoltaica possa ter viabilidade econômica para instalações conectadas à rede em telhados (Dávi et al., 2016). O desenvolvimento de pesquisas aplicadas, políticas favoráveis e incentivos financeiros são ações importantes para difundir ainda mais o uso da energia solar fotovoltaica.

Nesse contexto, é de extrema importância, a realização de estudos de viabilidade econômica de instalações fotovoltaicas próximas às cargas em redes de distribuição de baixa tensão (Vale et al., 2017). Nesses estudos é necessário averiguar a disponibilidade do recurso solar na área de interesse, pois a quantidade de energia produzida por um sistema fotovoltaico depende da irradiação solar no local onde é instalado (Villalva, 2015). Além disso, um gerador fotovoltaico tem suas características elétricas dependentes basicamente da irradiância e da temperatura nos módulos, sendo a influência da irradiância solar muito mais significativa do que a da temperatura, pois pode variar significativamente em curtos intervalos de tempo (Pinho e Galdino, 2014). Para isso, podem ser utilizados modelos matemáticos para se estimar a irradiação solar, ferramentas computacionais, dados de estações solarimétricas ou outros bancos de dados de irradiação solar.

Diante do exposto é possível se questionar sobre a influência que a escolha do banco de dados de irradiação solar exerce na análise econômica de um sistema solar fotovoltaico. Para responder este questionamento, esta pesquisa tem como objetivo comparar os resultados de análises econômicas de um sistema fotovoltaico realizadas com diferentes estimativas de geração de energia elétrica obtidas através da utilização de diferentes bancos de dados de irradiação solar.

\section{Materiais e Métodos}

O objeto de estudo desta pesquisa foi o sistema fotovoltaico instalado no telhado do campus Floriano do Instituto Federal do Piauí (IFPI), na cidade de Floriano, estado do Piauí. O sistema é composto por 660 módulos fotovoltaicos policristalinos com potência de $260 \mathrm{~W}$ cada, totalizando uma potência instalada de $171,6 \mathrm{kWp}$ e ocupa uma área de aproximadamente $1.122 \mathrm{~m}^{2}$ do telhado do 
campus e possui como coordenadas geográficas: Latitude $6^{\circ} 47^{\prime} 21,18^{\prime \prime}$ Sul e longitude $43^{\circ} 02^{\prime} 34,43^{\prime \prime}$ Oeste (Morais et al., 2019). Na Fig. 1 pode ser vista uma imagem aérea do sistema fotovoltaico. Este foi o primeiro Sistema Fotovoltaico Conectado à Rede (SFCR) de minigeração do estado do Piauí, e entrou em operação em junho de 2016 para atender parte da demanda de energia elétrica do campus.

Para a realização da pesquisa, primeiramente foi realizada a análise do recurso solar da cidade de Floriano-PI. Tendo em vista a não existência dos equipamentos de monitoramento do recurso solar instalado junto ao sistema fotovoltaico conforme orienta IEC 61724 (estação solarimétrica própria ou módulos de referência calibrados) (IEC, 1998) os dados de irradiação solar foram coletados em diversas bases de dados disponíveis para consulta pública. Foram consultados os seguintes bancos de dados: Projeto Solar and Wind Energy Resource Assessment (SWERA) (SWERA, 2017), Metadados da $2^{\mathrm{a}}$ edição do Atlas Brasileiro de Energia Solar (ABES) (Pereira et al., 2017) e dados provenientes de medições reais realizados pelo Instituto Nacional de Meteorologia (INMET) (Moura et al., 2011).

Para a coleta dos dados do Projeto SWERA foi escolhida a base de resolução moderada do Instituto Nacional de Pesquisas Espaciais (INPE), e obtidos os dados para a irradiação global horizontal para todos os meses do ano, através da seleção do local desejado pelas suas coordenadas geográficas. Os dados foram colhidos na plataforma no dia 16 de setembro de 2017 e referemse ao período de um ano completo. Já os dados disponibilizados pelo ABES, referem-se aos valores médios das estimativas do total diário de irradiação global horizontal, e foram coletados tendo em vista a localização geográfica do ponto de medição mais próximo do campus Floriano do IFPI. Os dados do INMET foram medidos através da estação meteorológica automática PI_A311, que possui como coordenadas geográficas: Latitude: $6^{\circ}$ 45'41,18'S e Longitude: $43^{\circ} 0^{\prime} 12,23^{\prime}$ ' O, e situa-se a uma distância de aproximadamente $1,46 \mathrm{~km}$ do campus Flo-

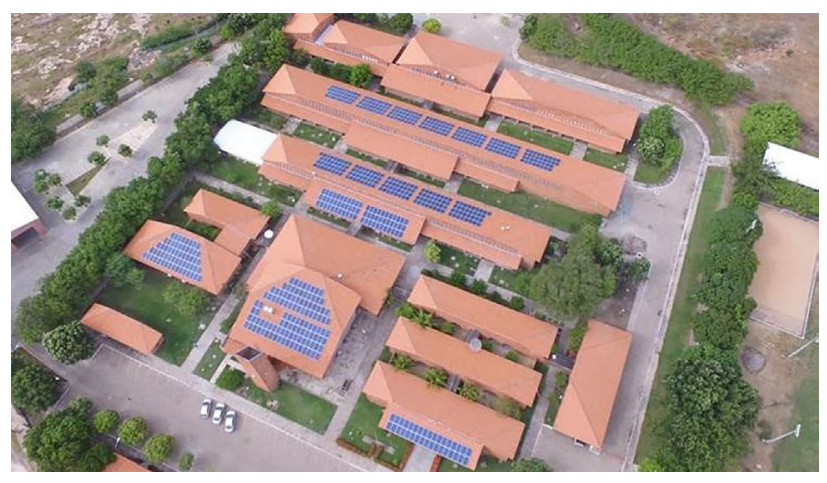

Figura 1 - Sistema fotovoltaico instalado sobre o telhado do campus Floriano do IFPI. Fonte: IFPI, 2019. riano do IPFI. Foram solicitados os dados relativos ao primeiro ano de operação do sistema fotovoltaico (1 de junho de 2016 a 31 de maio de 2017), via e-mail, pois já não se encontravam mais disponíveis para download no site oficial da instituição.

Recentemente os dados históricos anuais de todas as estações meteorológicas do Brasil foram disponibilizados para download site oficial do INMET através da plataforma do Banco de Dados Meteorológicos para Ensino e Pesquisa (BDMEP) (INMET, 2021). Vale a pena ressaltar que os dados relacionados ao período considerado se encontravam completos e medidos de hora em hora no período das 6:00 horas às 19:00 horas da noite, não necessitando de nenhuma técnica de gap filling para preenchimento de dados faltosos. De posse desses dados, foi estimada a produção de energia do sistema que, por fim, foram comparadas com a geração real do sistema. Os dados reais de geração do sistema fotovoltaico do campus Floriano do IFPI são relativos ao seu primeiro ano de operação. Tais dados foram obtidos através do software de monitoramento Sunny, próprio dos inversores instalados.

Conhecendo-se o modelo de módulo que será utilizado, pode-se estimar a quantidade de energia produzida pelo sistema na localidade que será instalado (Villalva, 2015), conforme está descrito na Eq. (1), na qual $E$ é a energia produzida mensalmente pelo SFCR, dada em Wh, $H$ é a irradiação solar diária no local de instalação do SFV, $\mathrm{em} \mathrm{Wh} / \mathrm{m}^{2} /$ dia, $A$ é a área da superfície do módulo, em $\mathrm{m}^{2}$, $\eta$ é a eficiência do módulo, $n$ é o número de módulos instalados, $d$ é a quantidade de dias do mês, e o valor 0,75 refere-se às perdas envolvidas no processo de geração fotovoltaica. Alguns fatores como eficiência do inversor, perdas de sincronismo e no circuito, perdas térmicas, por reflexão, entre outros, diminuem a quantidade de energia aproveitada pelo sistema. Para modelar essas perdas, baseado em estudos semelhantes (Macêdo e Zilles, 2007; Cabell e Pompermayer, 2013; EPE, 2012). Assim foi considerado um total de $25 \%$ de perdas.

$$
E=H \times A \times \eta \times n \times d \times 0,75
$$

As estimativas de geração obtidas foram também comparadas com as estimativas realizadas por dois softwares utilizados em projetos de instalações fotovoltaicas: Pvsyst e Solergo. Ambos os softwares utilizam bancos de dados de irradiação solar próprios. No PVsyst, a geração sintética de valores horários das médias mensais de irradiação solar é realizada por meio de modelos estocásticos desenvolvidos por Collares-Pereira na década de 80 (Mermoud et al., 2014). Além disso, inclui dados meteorológicos dos bancos de dados internacionais mais usados, como NASA, SSE RETScreen e Meteonorm (Muñoz et al., 2017). A ferramenta computacional Solergo permite avaliar sistemas $\mathrm{FV}$ conectados ou sistemas isolados à rede elétrica $\mathrm{O}$ 
software disponibiliza dados de irradiação proveniente do ABES e do banco de dados Electro Graphics (Monteiro Junior et al., 2016).

A geração de energia elétrica é proporcional à irradiação solar incidente no módulo fotovoltaico, onde nos meses de verão, com maior incidência solar, há maior geração de energia elétrica que nos meses de inverno, com menor incidência solar (Urbanetz Junior et al., 2014). Assim, analisando a Eq. (1), pode-se inferir que existe uma correlação linear entre a irradiação e a energia gerada pelo sistema fotovoltaico. Para avaliar o grau de correla- ção entre as estimativas de geração de energia calculadas e fornecidas pelos softwares, com os dados de irradiação solar medidos pela Estação Meteorológica PI_A311, foram calculados os coeficientes de correlação linear de Pearson que em seguida tiveram seu valor classificado em graus Forte, Moderado e Fraco. Este parâmetro pode ser calculado através da Eq. (2), na qual $r$ é o Coeficiente de Correlação de Pearson; $n$, o número de observações; $x i$, cada uma das médias mensais de irradiação solar global; $y i$ , cada uma das medições mensais de energia gerada pelo sistema.

$$
r=\frac{n \times \sum\left(x_{i} \times y_{i}\right)-\left(\sum x_{i}\right) \times\left(\sum y_{i}\right)}{\sqrt{\left[n \times \sum x_{i}{ }^{2}-\left(\sum x_{i}\right)^{2}\right]-\left[n \times \sum y_{i}{ }^{2}-\left(\sum y_{i}\right)^{2}\right]}}
$$

Quando duas variáveis são relacionadas estatisticamente de forma linear, o Coeficiente de Correlação Amostral ou Coeficiente de Correlação de Pearson $(r)$ é o instrumento adequado para medir o grau de intensidade da correlação entre as duas grandezas. Dessa maneira, podese determinar o grau de correlação entre os dados analisados com base nos seguintes parâmetros: se $0,8 \leq|r| \leq 1$, existe uma correlação aceitável entre as variáveis (correlação forte); se $0,5 \leq|r|<0,8$, existe uma correlação moderada entre as variáveis; e, por fim, se $0 \leq|r|<0,5$, não existe uma correlação aceitável entre as variáveis (correlação fraca) (Devore, 2014).

As estimativas de geração também foram submetidas a testes estatísticos de hipótese para a comparação de variáveis qualitativas, com o intuito de se atestar o seu grau de precisão das estimativas realizadas com os dados reais de geração, e verificar a diferença entre elas. Os testes de hipóteses, também conhecidos como testes de significância, são utilizados quando se tem alguma ideia ou conjectura sobre o comportamento de uma variável ou de uma possível associação entre variáveis. Servem para verificar se os dados fornecem evidência suficiente para que se possa aceitar como verdadeira uma hipótese de pesquisa, precavendo-se com certa segurança, de que as diferenças observadas nos dados não são meramente casuais (Barbetta, 2019).

Assim, o propósito dos testes de hipóteses não é de questionar valores das estatísticas da amostra, mas fazer um julgamento sobre a diferença entre dois resultados estatísticos ou entre a estatística da amostra e um parâmetro hipotético da população (Tondolo e Schneider, 2006). Dentre os diversos testes de hipóteses tem-se o Teste-F que é utilizado para saber se duas variâncias populacionais são estatisticamente iguais ou se uma é maior do que a outra, e o Teste-T, que, por sua vez, é utilizado quando se quer comparar a média das populações (Tavares, 2007). Tendo sido escolhido qual estatística irá sustentar o teste, devem ser adotados os procedimentos descritos na Tabela 1.

A aceitação ou rejeição da hipótese nula está condicionada à significância da probabilidade calculada pelo teste, que no caso desta pesquisa foi considerado como sendo $\alpha=0,05$. Se a probabilidade for superior ao nível de significância desejado, a hipótese nula é aceita e, por outro

Tabela 1 - Procedimentos do teste de hipóteses.

\begin{tabular}{ll}
\hline Procedimentos & \multicolumn{1}{c}{ Descrição } \\
\hline $\begin{array}{l}\text { Formular a hipótese nula }\left(H_{0}\right) \\
\begin{array}{l}\text { Selecionar o nível de sig- } \\
\text { nificância }(\alpha)\end{array}\end{array}$ & $\begin{array}{l}\text { A hipótese nula é necessária para testar estatisticamente a significância. } \\
\text { A escolha do nível de significância deve ser feita antes da coleta de dado. O nível de significância está relacionado ao } \\
\text { risco } \alpha \text { que se deseja aceitar. }\end{array}$ \\
$\begin{array}{l}\text { Computar o valor da diferença } \\
\text { calculada }\end{array}$ & $\begin{array}{l}\text { Depois de coletar os dados, utilizar a fórmula do teste escolhido e obter o valor calculado. } \\
\text { Obter o valor crítico de teste }\end{array}$ \\
$\begin{array}{l}\text { Interpretar o teste } \\
\text { Após obter o valor calculado, deve-se procurar o valor crítico na tabela correspondente. }\end{array}$ \\
$\begin{array}{l}\text { Se o valor calculado for maior que o valor crítico, rejeita-se a hipótese nula. Complementarmente, se o valor calculado } \\
\text { for menor que o valor de tabela, aceita-se a hipótese nula. }\end{array}$
\end{tabular}


lado, se a probabilidade calculada for inferior, a hipótese nula é rejeitada (Tondolo e Schneider, 2006) sabendo que se o valor calculado da estatística foi menor do que o tabelado (crítico), então, ele caiu na região de aceitação da hipótese nula (Tavares, 2007). Assim, para determinar se existe ou não diferença significativa entre as estimativas de geração realizadas e os dados reais de geração do sistema, inicialmente realizou-se o Teste-F para a análise das variâncias entre cada estimativa realizada individualmente e os dados reais de geração, considerando a hipótese inicial nula, ou seja, as variâncias são iguais, para em seguida ser realizado do Teste-T correspondente, ou seja, variâncias iguais ou diferentes, para a análise das médias das estimativas realizadas. Para ambos os testes estatísticos, foi utilizado o Microsoft Excel. Na Tabela 2 encontram-se descritos os procedimentos necessários para interpretar os resultados dos Testes $\mathrm{F}$ e $\mathrm{T}$ utilizando-se o software Microsoft Excel (Bernal e Silva, 2012).

Vale a pena ressaltar que a suposição a normalidade dos dados é exigida para a realização de muitos métodos de inferência estatística, como Correlação Linear de Pearson, Regressão Linear e Testes de hipóteses (Torman

Tabela 2 - Procedimentos para a interpretação dos resultados no Excel.

\begin{tabular}{|c|c|}
\hline Teste F & Interpretação dos resultados \\
\hline$F \leq 1$ & $\begin{array}{l}H_{0}: \sigma_{1}^{2}=\sigma_{2}^{2} \text { e } H_{1}: \sigma_{1}^{2}<\sigma_{2}^{2} \\
\text { Unicaudal à esquerda }\end{array}$ \\
\hline$F>1$ & $\begin{array}{l}H_{0}: \sigma_{1}^{2}=\sigma_{2}^{2} \text { e } H_{1}: \sigma_{1}^{2}>\sigma_{2}^{2} \\
\text { Unicaudal a direita }\end{array}$ \\
\hline$P(F \leq f)=\alpha_{d}$ & $\begin{array}{l}\alpha_{d}<\alpha \Rightarrow \text { rejeição de } H_{0} \\
\alpha_{d}>\alpha \Rightarrow \text { não rejeição de } H_{0}\end{array}$ \\
\hline$F$ e Fcrítico Unicaudal à esquerda & $\begin{array}{l}F<F \text { crítico } \Rightarrow \text { rejeição de } H_{0} \\
F>F \text { crítico } \Rightarrow \text { não rejeição de } H_{0}\end{array}$ \\
\hline$F$ e Fcrítico Unicaudal à direita & $\begin{array}{l}F>\text { Fcrítico } \Rightarrow \text { rejeição de } H_{0} \\
F<F c r i ́ t i c o \Rightarrow \text { não rejeição de } H_{0}\end{array}$ \\
\hline Teste $\mathrm{T}$ & Interpretação dos resultados \\
\hline Stat $t<0$ & $\begin{array}{l}H_{0}: \mu_{1}=\mu_{2} \text { e } H_{1}: \mu_{1}<\mu_{2} \\
\text { Unicaudal à esquerda }\end{array}$ \\
\hline Stat $t>0$ & $\begin{array}{l}H_{0}: \quad \mu_{1}=\mu_{2} \text { e } H_{1}: \mu_{1}>\mu_{2} \\
\text { Unicaudal à direita }\end{array}$ \\
\hline$P(T \leq t)=\alpha_{d}$ & $\begin{array}{l}\alpha_{d}<\alpha \Rightarrow \text { rejeição de } H_{0} \\
\alpha_{d}>\alpha \Rightarrow \text { não rejeição de } H_{0}\end{array}$ \\
\hline $\begin{array}{l}\text { Stat } t \text { e tcrítico Unicaudal à } \\
\text { esquerda }\end{array}$ & $\begin{array}{l}\text { Stat } t<- \text { tcrítico } \Rightarrow \text { rejeição de } H_{0} \\
\text { Stat } t>- \text { tcrítico } \Rightarrow \text { não rejeição de } \\
H_{0}\end{array}$ \\
\hline Stat $t$ e tcrítico Unicaudal à direita & $\begin{array}{l}\text { Stat } t>\text { tcrítico } \Rightarrow \text { rejeição de } H_{0} \\
\text { Stat } t<\text { tcrítico } \Rightarrow \text { não rejeição de } \\
H_{0}\end{array}$ \\
\hline
\end{tabular}

Fonte: Adaptado de Bernal e Silva (2012). et al., 2012). É importante verificar, antes das análises estatísticas, que qualquer uma das hipóteses incorridas em testes individuais não seja violada, pois assumir a normalidade sem a realização de testes para verificação, conforme sua conveniência pode fazer com que a interpretação, compreensão e inferência dos dados tornam-se inválidas (Machado et al., 2015). Para a avaliação da normalidade de um conjunto de dados podem ser analisados o seu histograma, afim de se identificar grandes assimetrias e descontinuidades dos dados, e os Diagramas Q-Q (QuantilQuantil) onde, idealmente, se apresentar como uma linha diagonal caso os dados sejam próximos à distribuição normal (Miot, 2017).

Nesta pesquisa, a normalidade dos dados de irradiação solar mensal foi verificada utilizando os diagramas Q$\mathrm{Q}$, relacionando cada um dos bancos de dados com os dados medidos pelo INMET, como pode ser visto na Fig. 2, juntamente com as linhas de base, demostrando a normalidade dos dados.

Os dados medidos pelo INMET, por sua vez, tiveram sua normalidade verificada através do histograma dos totais diários, considerando o período de geração do primeiro ano de operação, juntamente com a curva de distribuição normal dos dados, como pode ser visto na Fig. 3.

A viabilidade econômica de um projeto pode ser determinada mediante uma perspectiva qualitativa, na qual o investimento provém da manifestação de interesses, sem considerar os custos ou benefícios da sua implantação, ou de uma perspectiva quantitativa, onde se realiza uma análise econômico financeira, através da apuração de métricas próprias da Engenharia Econômica nas quais se projeta um fluxo de caixa com entradas e saídas e capital e periodicidade (Frezatti, 2008). Nesta pesquisa foi considerada a análise quantitativa com as seguintes métricas: Valor Presente Líquido (VPL), Taxa Interna de Retorno (TIR), Payback (PB) e Relação Custo Benefício (RCB).

O VPL de um investimento é a diferença entre os benefícios gerados pelo projeto e seus custos, onde os fluxos de caixa são trazidos a um valor presente descontados a uma determinada taxa. Se o VPL for positivo, o projeto é considerado economicamente viável, pois as receitas superam o valor investido somado às despesas (Nakabayashi, 2015). A expressão para o cálculo do VPL é mostrada na Eq. (3), em que $F_{c t}$ é o fluxo de caixa no período $t$ considerado, $r$ é a taxa de desconto, $\mathrm{n}$ é o horizonte de vida útil do projeto e $I_{0}$ é o investimento inicial.

$$
V P L=\sum_{t=1}^{n} \frac{F_{c t}}{(1+r)^{t}}-I_{0}
$$

A TIR é o valor que, quando empregado como a taxa de desconto no cálculo do VPL, faz com que seu valor fique igual a zero. Assim, é a taxa que remunera o investimento. Se a TIR for maior do que a Taxa de Desconto, o projeto 

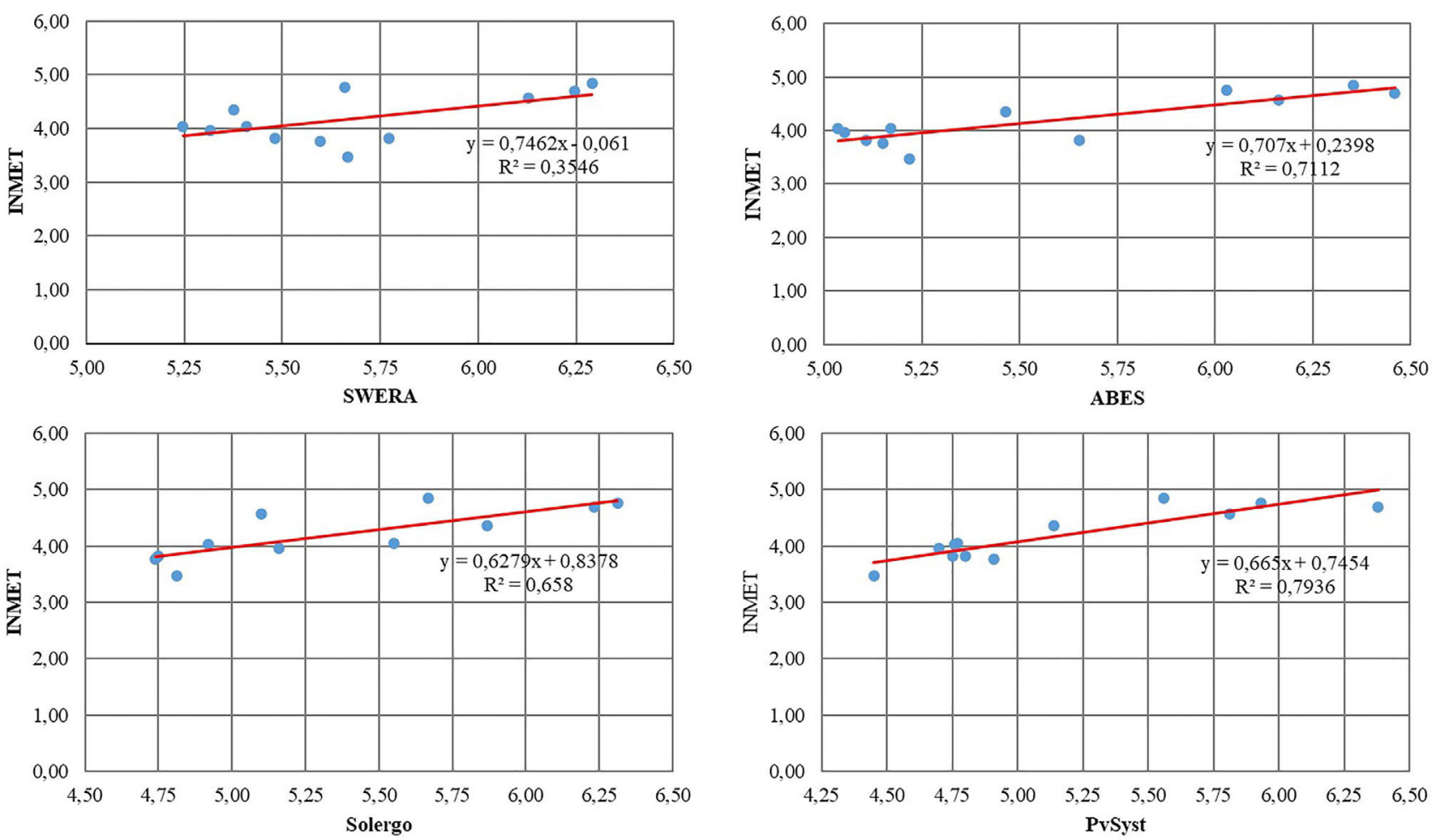

Figura 2 - Linhas base dos bancos de dados de irradiação solar utilizados na pesquisa.

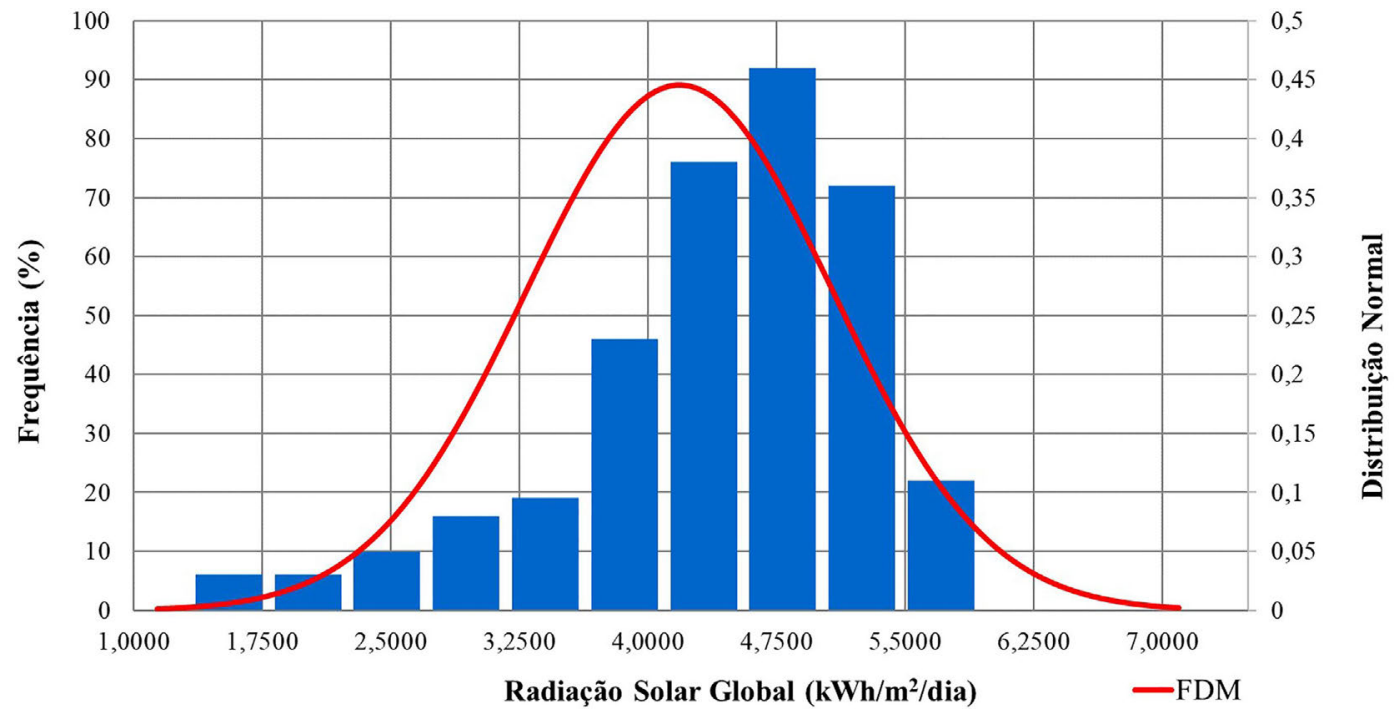

Figura 3 - Histograma e Curva de Distribuição Normal dos dados medidos pelo INMET.

pode ser aceito (Silva et al., 2019). Eq. (4) demonstra a relação entre a TIR e o VLP.

$$
V P L=\frac{F_{c 1}}{(1+T I R)^{1}}+\cdots+\frac{F_{c n}}{(1+T I R)^{n}}-I_{0}=0
$$

$\mathrm{O} \mathrm{PB}$, ou tempo de retorno de um investimento, é número de períodos necessários para que o fluxo de caixa acumulado se torne positivo, isto considerando que este fluxo é do tipo em que o investimento é realizado no primeiro período e as receitas estão nos anos seguintes (Nakabayashi, 2015). Pode ser calculado por meio da Eq. (5), em que

$$
P B=\frac{I_{0}}{R_{e}}
$$

A RCB é um indicador que serve para selecionar um entre vários projetos quando o capital é limitado. Devem ser escolhidos projetos que apresentem uma relação menor 
que um, pois quanto menor a RCB, melhor (Silva et al., 2019) e pode ser determinada através da Eq. (6).

$$
R C B=\frac{I_{0}}{V P L}
$$

\section{Resultados e Discussão}

Na Fig. 4, é apresentada a variação mensal da Irradiação Solar Global Horizontal ao longo do período analisado, referente ao banco de dados do projeto SWERA, ao ABES e aos dados reais medidos pela estação automática PI_A311 do INMET.

Analisando o gráfico, destaca-se, no caso do projeto SWERA, o período que vai de setembro a dezembro pois apresenta valores médios de irradiação solar global acima da média anual $\left(5,68 \mathrm{kWh} / \mathrm{m}^{2} /\right.$ dia $)$. No caso dos dados do ABES, os meses com irradiação solar acima da média anual de $5,57 \mathrm{kWh} / \mathrm{m}^{2} /$ dia, são os meses entre agosto e dezembro. Em contrapartida, no caso dos dados medidos pelo INMET, percebe-se que o período onde os níveis médios de irradiação solar global superam a média anual $\left(4,18 \mathrm{kWh} / \mathrm{m}^{2} / \mathrm{dia}\right)$ vai de julho a novembro. Ademais, nota-se que em todos o período analisado, os valores de irradiação solar global referentes ao projeto SWERA e ao ABES são maiores que os dados reais medidos pela estação PI_A311 do INMET, porém, apresenta valores bem próximos quando analisados entre si. De acordo com Stedile et al. (2018) isso pode ocorrer porque os dados coletados pelos pirômetros das estações do INMET estão no plano horizontal, o que pode diminuir o seu valor, quando considerado o plano inclinado nos qual os módulos fotovoltaicos estejam instalados.
Entretanto, percebe-se que existem indicativos de condições favoráveis para o aproveitamento da energia solar no Estado do Piauí. No entanto, é preciso que se avalie o quão eficiente será esse aproveitamento, tendo como base a realização de estudos de viabilidade técnica e financeira (Lira, 2015). Diante disso, são apresentadas na Tabela 3, as estimativas calculadas utilizando os dados de irradiação do projeto SWERA, do ABES e os dados de medição do INMET, conforme encontra-se descrito na Eq. (1), as estimativas fornecidas pelos softwares Solergo e PVsyst, bem como a geração real do sistema fotovoltaico.

O sistema fotovoltaico gerou no seu primeiro ano de operação um total de $256.218,84 \mathrm{kWh}$, perfazendo uma média mensal de $21.351,57 \mathrm{kWh}$. A estimativa de geração utilizando os dados de irradiação do projeto SWERA deu um total anual de $266.836,16 \mathrm{kWh}$ com uma média mensal de $22.236,35 \mathrm{kWh}$. Esses valores superam a geração real do sistema em 4,14\%. Já a estimativa realizada utilizando os dados fornecidos pelo ABES trouxe um total anual de $261.726,73 \mathrm{kWh}$ com uma média de $21.810,56 \mathrm{kWh}$ que superam a geração real em 2,15\%. Em contrapartida, a estimativa de geração utilizando os dados medidos pelo INMET acusou um total anual de 196.456,98 kWh com um média mensal de $16.371,41 \mathrm{kWh}$, ou seja, valores bem próximos da geração real com uma diferença de aproximadamente $23,32 \%$ acima.

No caso das estimativas apresentadas pelos softwares, a estimativa realizada utilizando o Solergo mostrou um total anual gerado de 248.862,22 kWh com uma média mensal de 20.738,52 kWh. Esta estimativa apresenta valores abaixo da geração real do sistema com uma diferença de $2,87 \%$ menor. Já a estimativa feita pelo PVsyst apresentou um total anual de $270.717,00 \mathrm{kWh}$, com uma

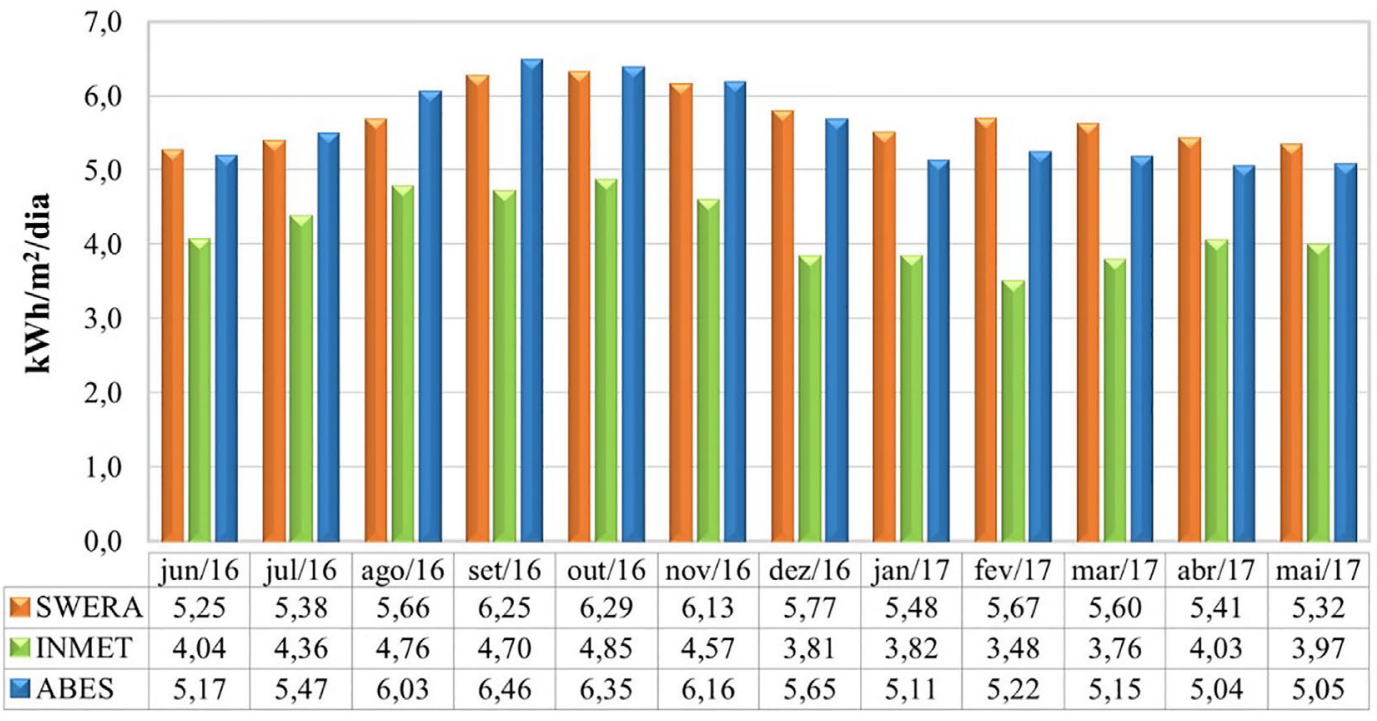

Figura 4 - Variação da irradiação global horizontal para a cidade de Floriano. 
Tabela 3 - Estimativas de geração de energia elétrica e geração real (kWh).

\begin{tabular}{|c|c|c|c|c|c|c|}
\hline Mês & SWERA & INMET & ABES & Solergo & PVsyst & Real \\
\hline jun/16 & $20.253,69$ & $15.598,47$ & $19.960,33$ & $22.406,60$ & $22.950,00$ & $19.676,44$ \\
\hline jul/16 & $21.439,37$ & $17.374,87$ & $21.798,36$ & $24.401,07$ & $25.501,00$ & $22.084,85$ \\
\hline ago/16 & $22.576,16$ & $18.990,30$ & $24.051,99$ & $25.698,64$ & $28.332,00$ & $24.871,22$ \\
\hline set/16 & $24.109,89$ & $18.134,53$ & $24.935,94$ & $23.878,14$ & $27.783,00$ & $24.167,30$ \\
\hline out/16 & $25.093,04$ & $19.337,32$ & $25.340,34$ & $21.941,73$ & $23.554,00$ & $25.789,24$ \\
\hline nov/16 & $23.658,26$ & $17.655,88$ & $23.789,50$ & $18.827,07$ & $22.552,00$ & $23.014,24$ \\
\hline dez/16 & $23.026,88$ & $15.212,98$ & $22.544,25$ & $18.025,01$ & $18.653,00$ & $20.147,88$ \\
\hline $\mathrm{jan} / 17$ & $21.862,18$ & $15.244,89$ & $20.374,39$ & $18.094,07$ & $19.155,00$ & $19.478,08$ \\
\hline $\mathrm{fev} / 17$ & $20.416,59$ & $12.523,04$ & $18.795,37$ & $16.765,24$ & $16.758,00$ & $16.979,34$ \\
\hline $\operatorname{mar} / 17$ & $22.320,88$ & $15.005,57$ & $20.545,90$ & $18.588,12$ & $21.467,00$ & $19.504,28$ \\
\hline $\mathrm{abr} / 17$ & $20.875,16$ & $15.559,87$ & $19.435,36$ & $19.071,46$ & $21.174,00$ & $20.490,78$ \\
\hline $\mathrm{mai} / 17$ & $21.204,04$ & $15.819,27$ & $20.155,01$ & $21.165,06$ & $22.838,00$ & $20.015,19$ \\
\hline Total & $266.836,16$ & $196.456,98$ & $261.726,73$ & $248.862,22$ & $270.717,00$ & $256.218,84$ \\
\hline Média & $22.236,35$ & $16.371,41$ & $21.810,56$ & $20.738,52$ & $22.559,75$ & $21.351,57$ \\
\hline
\end{tabular}

média mensal de 22.559,75 kWh. Estes valores estão acima da geração real do sistema com uma diferença de $5,66 \%$.

Um estudo realizado com 18 softwares fotovoltaicos comparou as estimativas realizadas com três plantas fotovoltaicas reais, onde foram descobertas diferenças evidentes que chegavam a até 20\% (Podewils, 2011). Verificou-se que o software Solergo em comparação com os demais, apresentou simulações em tempo real com as melhores precisões. Em média, a previsão se desvia em $3 \%$ do rendimento real. O software PVsyst está entre os mais conhecidos no setor fotovoltaico, porém apresentou um desvio médio de $6,2 \%$. Dessa maneira, os valores encontrados nesta pesquisa para as simulações realizadas pelos softwares, estão de acordo com os dados informados.

Um resumo das estimativas de geração realizadas pode ser visto na Tabela 4 juntamente com o percentual de produção em relação à geração real do sistema. Verifica-se que as estimativas fornecidas pelos softwares e a estimativa calculada utilizando os dados do Projeto SWERA e do ABES se aproximaram bem dos valores reais do sistema, quando comparadas com as estimativas calculadas utilizando os dados do INMET.

Tabela 4 - Resumo das estimativas de geração de energia em kWh.

\begin{tabular}{lccc}
\hline Base & Total anual & Média mensal & Porcentagem \\
\hline INMET & $196.456,98$ & $16.371,41$ & $23,32 \%$ abaixo \\
Solergo & $248,862,22$ & $20.738,52$ & $2,87 \%$ abaixo \\
ABES & $261.726,73$ & $21.810,56$ & $2,14 \%$ acima \\
SWERA & $266.836,16$ & $22.236,35$ & $4,14 \%$ acima \\
PVsyst & $270.717,00$ & $22.559,75$ & $5,66 \%$ acima \\
\hline
\end{tabular}

Os dados de irradiação do projeto SWERA, do ABES, os dados obtidos com o INMET e a geração real do sistema foram colocados em um mesmo gráfico. Também foram colocados os dados de irradiação fornecidos pelos softwares usados nesta pesquisa para estimar a energia gerada pelo sistema (Fig. 5).

Analisando o gráfico da geração real do $1^{\circ}$ ano de operação do sistema, com o perfil dos gráficos dos índices de radiação do INMET, verifica-se que visualmente, os mesmos apresentam um perfil semelhante. Fato que não se verifica ao fazer a mesma comparação com os dados de irradiação do projeto SWERA e do ABES. Com relação às estimativas apresentadas pelos softwares analisados, verifica-se que a estimativa apresentada pelo Solergo também apresenta perfil semelhante ao da geração real do sistema. Assim, foi determinado o Coeficiente de Correlação de Pearson entre cada banco de dados de irradiação, inclusive os fornecidos pelos softwares, com a geração real do sistema fotovoltaico. Os resultados encontram-se descritos na Tabela 5 .

A análise de correlação apresentada mostra que existe uma correlação moderada entre os dados mensais de irradiação fornecidos pelo banco de dados do projeto SWERA $(r=0,64)$ e pelo software Solergo $(r=0,76)$ com

Tabela 5 - Resultados da Análise de Correlação.

\begin{tabular}{lcc}
\hline Estimativa & Coeficiente & Correlação \\
\hline SWERA & 0,64 & Moderada \\
Solergo & 0,76 & Moderada \\
ABES & 0,86 & Forte \\
PVsyst & 0,87 & Forte \\
INMET & 0,98 & Forte \\
\hline
\end{tabular}




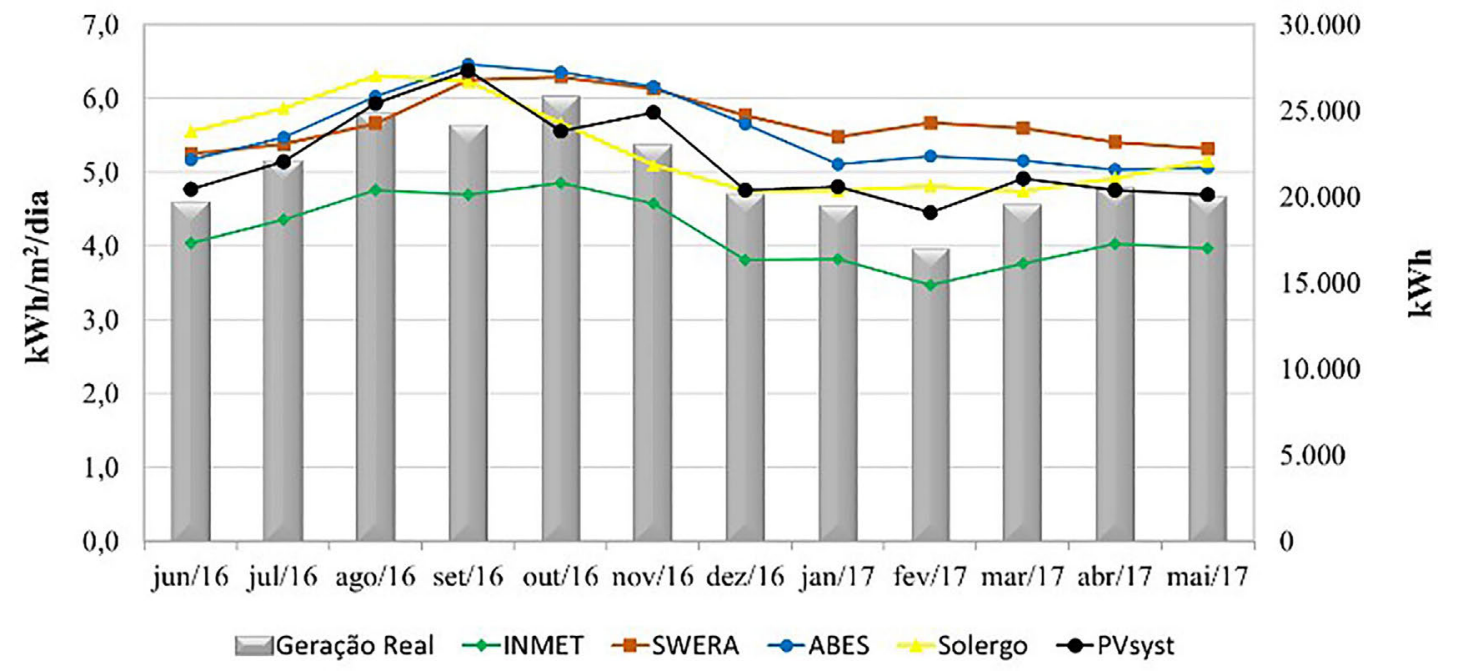

Figura 5 - Perfil das curvas de irradiação solar e geração do SFCR.

os dados mensais de geração real do sistema fotovoltaico. Por outro lado, os dados de irradiação solar fornecidos pelo INMET, pelo software PVsyst e pelo ABES apresentaram uma correlação forte com os dados reais de geração do sistema $(r=0,98, r=0,86$ e $r=0,87$, respectivamente). Porém, os dados fornecidos pelo INMET apresentaram uma correlação bem mais alta, muito próxima da unidade. Analisando-se somente esse parâmetro poder-se-ia sugerir que a utilização dos dados de irradiação fornecidos por estações meteorológicas do INMET próximas ao sistema fotovoltaico é suficiente para se estimar com proximidade a geração de um sistema fotovoltaico. Daí a necessidade da realização dos testes de hipóteses.

A seguir são apresentados os resultados dos testes de hipóteses realizados entre as estimativas de geração e a geração real do sistema fotovoltaico. Também foram realizadas entre as estimativas fornecidas pelos softwares analisados nesta pesquisa. Tendo como hipótese inicial que não existe diferença significativa entre cada estimativa de geração, utilizando os bancos de dados da pesquisa, e a geração real do sistema. Primeiramente realizou-se o Teste-F para que, dependendo dos resultados de cada análise, proceder para o Teste-T correspondente. Em todos os casos, com exceção dos dados medidos pelo INMET, confirmou-se a hipótese inicial nula deste teste, ou seja, as variâncias são iguais. Além disso, em todos os testes o nível de significância da probabilidade calculada $P$, foi maior que o nível significância requerido para o teste $(\alpha=0,05)$, o que reforça a hipótese inicial nula. Os resultados do Teste-F encontram-se descritos na Tabela 6. A variância dos dados de geração real é de 6.921.090,82.

Dessa forma, procedeu-se agora com o Teste-T, para análise das médias entre cada estimativa realizada individualmente e os dados reais de geração, considerando-se a hipótese inicial nula das médias serem iguais. Também
Tabela 6 - Resultados do Teste-F.

\begin{tabular}{lcccc}
\hline Estimativa & Variância & $F$ & $P=\alpha_{d}$ & $F$ crítico \\
\hline INMET & $3.842 .540,01$ & 0,56 & 0,17 & 0,36 \\
SWERA & $2.306 .940,55$ & 0,33 & 0,04 & 0,36 \\
ABES & $5.115 .011,52$ & 0,74 & 0,31 & 0,36 \\
Solergo & $8.498 .774,77$ & 1,23 & 0,37 & 2,82 \\
PVsyst & $12.190 .927,30$ & 1,76 & 0,18 & 2,82 \\
\hline
\end{tabular}

foram consideradas as estimativas fornecidas pelos softwares. Concluiu-se, de acordo com as orientações da Tabela 2, que a média das estimativas de geração feitas com os bancos de dados realizados nesta pesquisa, à exceção dos dados medidos pelo INMET, possui diferença significativa com a geração real do sistema. Os resultados do Teste-T encontram-se descritos na Tabela 7, sendo a média dos dados de geração real igual a 21.351,57.

Dessa maneira, os valores encontrados nas análises estatísticas mostram que a energia gerada por sistemas fotovoltaicos pode ser estimada utilizando a Eq. (1), utilizando os bancos de dados utilizados nesta pesquisa. Porém, os dados fornecidos pelas estações meteorológicas, mesmo tendo altas correlações e não possuindo diferenças significativas com a geração real, não são os dados ideais para que seja estimada a energia a ser gerada por um sistema fotovoltaico, pelo fato de traduzirem apenas medições de um período específico, não retratando a realidade de períodos mais logos, como no caso dos bancos de dados provenientes de séries históricas. Por outro lado, a metodologia de estimação de valores pode ser utilizada por pesquisadores e projetistas em análises econômicas de sistemas fotovoltaicos, por trazer valores próximos da realidade. A utilização de dados fornecidos por softwares de simulação são outras possíveis alternativas para se estimar a geração de um sistema fotovoltaico, tendo em vista que os resultados se aproximam bem dos valores reais gerados 
Tabela 7 - Resultados do Teste T.

\begin{tabular}{lcccc}
\hline Estimativa & Média & stat $t$ & $P=\alpha_{d}$ & Tcrítico \\
\hline INMET & $16.371,42$ & $-5,26$ & $1,41 \times 10^{-5}$ & 1,72 \\
SWERA & $22.236,35$ & 1,01 & 0,16 & 1,73 \\
ABES & $21.810,56$ & 0,46 & 0,33 & 1,72 \\
Solergo & $20.738,52$ & $-0,54$ & 0,30 & 1,72 \\
PVsyst & $22.559,75$ & 0,96 & 0,17 & 1,72 \\
\hline
\end{tabular}

pelos sistemas. Dessa forma, informações como rendimento do sistema fotovoltaico e economia conseguida anualmente poderão ser fornecidas por softwares projetados para simular especificamente sistemas fotovoltaicos com grandes precisões (Podewils, 2011)

A seguir são apresentados os resultados das análises econômicas feitas utilizando-se as estimativas de geração de energia. Na Tabela 8 encontra-se descrito o resultado da análise econômica do sistema fotovoltaico do campus Floriano do IFPI. É comum considerar a degradação na geração dos módulos fotovoltaicos ao longo do tempo, a qual geralmente é considerada, nos modelos de projeção, com valores de $0,5 \%$ a $1 \%$ ao ano, embora valores mais baixos sejam alcançados na prática (Nakabayashi, 2015). Assim, foi considerada uma taxa de degradação anual de $0,8 \%$ ao ano, na produção de energia (perda total de $20 \%$ em 25 anos segundo o fabricante). $\mathrm{O}$ investimento inicial foi de $\mathrm{R} \$ 1.150 .000,00$ e $1 \%$ desse valor aplicado a cada 10 anos, referente à troca dos inversores. Como benefício do projeto foi considerado o valor de $\mathrm{R} \$ 0,331276 / \mathrm{kWh}$ a ser economizado nas faturas de energia. A taxa de des-

Tabela 8 - Análise econômica com dados de geração real.

\begin{tabular}{lc}
\hline Dados da análise & Valor \\
\hline Economia de Energia Anual & $256.218,84 \mathrm{kWh}$ \\
Economia Percentual de Energia Anual & $59,01 \%$ \\
Economia Monetária de Energia Anual & $\mathrm{R} \$ 84.879,15$ \\
Taxa Interna de Retorno & $1,91 \%$ \\
Valor Presente Líquido & $\mathrm{R} \$ 263.172,06$ \\
Relação Custo Benefício & 4,37 \\
Payback & 19 anos \\
\hline
\end{tabular}

conto do fluxo de caixa foi de $1,52 \%$, dada pela diferença entre a média dos valores anuais da Taxa Selic e a média dos valores de reajuste anual das tarifas de energia informados pela Aneel, ambas observadas no período de 2008 a 2017 (Morais et al., 2019).

Com base nessa análise, o valor investido inicialmente retornará em 19 anos, e, após os 25 anos de vida útil do projeto, o VPL foi de R\$263.172,06, a uma TIR de $1,91 \%$ ao ano. O que representa uma economia anual de $59,01 \%$ considerando a geração do primeiro ano de operação do sistema que foi de $434.192 \mathrm{kWh}$. A RCB foi de 4,37. Na Tabela 9 são apresentados os resultados da análise econômica utilizando as estimativas de energia obtida com os dados de irradiação solar do projeto SWERA, INMET, ABES e Solergo e PVsyst.

Analisando os resultados com os dados do Projeto SWERA, percebe-se que o payback será 18 anos e após os 25 anos de vida útil do projeto, o VPL deverá ser de $\mathrm{R} \$ 329.355,06$, a uma TIR de $2,36 \%$ ao ano. O que representa uma economia anual de $61,46 \%$. A RCB foi de 3,49 . Já com a estimativa utilizando os dados medidos do INMET, percebe-se que o valor investido inicialmente não retornará dentro do tempo de vida útil do projeto, devendo vir apenas aos 29 anos, sendo que no seu último ano, VPL será de R \$ 109.352,97, a uma TIR de $-0,87 \%$ ao ano. No entanto, o projeto ainda trará uma economia anual de $196.456,98 \mathrm{kWh}$ de energia. O que representa uma economia anual de $45,25 \%$. A RCB neste foi de $-10,52$, o que mostra que o projeto terá mais gastos do que retorno. Utilizando os dados do ABES para as estimativas, o investimento inicial retornará em 18 anos e após os 25 anos de vida útil do projeto, o VPL foi de R $\$ 297.505,52$ a uma TIR de $2,14 \%$ ao ano. O que representa uma economia anual de $60,25 \%$. A RCB foi de 3,87 .

As estimativas feitas utilizando o software Solergo demostram um payback de 18 anos e após a vida útil do projeto, o VPL será de R $\$ 217.314,69$ a uma TIR de $1,59 \%$ ao ano. O que representa uma economia anual de $57,32 \%$. A RCB foi de 5,29. Por fim, os resultados da análise econômica utilizando a estimativa de energia obtida através do software PVsyst, demonstram que que haverá retorno financeiro após 17 anos. E ao final do tempo de

Tabela 9 - Análise Econômica das estimativas realizadas na pesquisa.

\begin{tabular}{|c|c|c|c|c|c|}
\hline Análise & SWERA & INMET & ABES & Solergo & PVsyst \\
\hline Economia de Energia Anual (kWh) & $266.836,16$ & $196.456,98$ & $261.726,73$ & $248.862,22$ & $270.717,00$ \\
\hline Economia Percentual de Energia Anual (\%) & 61,46 & 45,25 & 60,28 & 57,32 & 62,35 \\
\hline Economia Monetária de Energia Anual (R\$) & $88.396,41$ & $65.081,48$ & $86.703,79$ & $82.442,08$ & $89.682,04$ \\
\hline Taxa Interna de Retorno (\%) & 2,36 & $-0,87$ & 2,14 & 1,59 & 2,52 \\
\hline Valor Presente Líquido (R\$) & $329.355,06$ & $-109.352,97$ & $297.505,52$ & $217.314,69$ & $353.546,28$ \\
\hline Relação Custo Benefício & 3,49 & $-10,52$ & 3,87 & 5,29 & 3,25 \\
\hline Payback (anos) & 18 & 29 & 18 & 19 & 17 \\
\hline
\end{tabular}


vida útil, o VPL será de R\$353.546,58, a uma TIR de $2,52 \%$. Isso trará uma economia anual de energia de $62,35 \%$, ou seja, $270,717,00 \mathrm{kWh}$. Neste caso a RCB foi de 3,25 anos. Pode-se perceber que os parâmetros das estimativas de geração de energia que se aproximaram da geração real, também apresentam análises econômicas aproximadas, porém com valores distintos para cada parâmetro.

\section{Conclusões}

Foi verificado que os bancos de dados de irradiação ou softwares que utilizam séries históricas para a sintetização dos dados fornecidos, são suficientes para se estimar com precisão adequada a geração anual do sistema fotovoltaico, através da metodologia de cálculo, descrita pela Equação 1, sendo alternativas viáveis a serem utilizadas nas análises de viabilidade econômica tanto no caso de instalações de novos sistemas quanto com vistas a replicação de sistemas fotovoltaicos existentes, através das métricas utilizadas na engenharia econômica, tais como Valor Presente Líquido, Taxa Interna de Retorno, Payback e Relação Custo-Benefício, por apresentar uma estimativa precisa e coerente com a realidade. Dentre os bancos de dados de irradiação solar apresentados nesta pesquisa, o que se mostrou mais eficaz foi o banco disponível no ABES. No caso dos softwares estudados, o Pvsyst foi o que se mostrou mais eficaz.

A escolha da metodologia para o cálculo das estimativas de geração pode influenciar diretamente nos resultados das análises de viabilidade, podendo levar os gestores a não optarem na realização de um determinado projeto ou até mesmo a optarem por uma opção ruim, tendo em vista possíveis resultados que lhes são apresentados. Assim, tendo em vista a dimensão do erro gerado pela metodologia escolhida, alguns problemas podem surgir, como consequência associada, no decorrer da utilização do sistema. Problemas estes que poderão ser evidenciados a curto, médio e longo prazo.

\section{Referências}

ANEEL, Agência Nacional de Energia Elétrica. Resolução no 482, de 17 de abril de 2012. Brasília, 2012.

ANEEL, Agência Nacional de Energia Elétrica. Resolução $\mathbf{n}^{\mathbf{0}}$ 687, de 24 de novembro de 2015. Brasília, 2015.

BASHIR, R.; MAKHDOOM, A.R.; BILAL, M.K.; BADAR, M.A. Comparative study of the photovoltaic behavior of ruthenium and the other organic and inorganic Dye-Sensitized Solar Cells (DSSC). Optik, v. 157, p. 11-15, 2018.

BERNAL, R.; SILVA, N.N. O Uso do Excel Para Análises Estatísticas: Curso de Bioestatística. São Paulo: Universidade de São Paulo, p. 50, 2012,

CABELlO, A.F.; POMPERMAYER, F.M. Energia Fotovoltaica Ligada à Rede Elétrica: Atratividade Para o Consumidor Final e Possíveis Impactos no Sistema Elé- trico. Rio de Janeiro: Instituto de Pesquisa Econômica Aplicada, p. 50, 2013.

DÁVI, G.A.; CAAMAÑO-MARTÍN, E.; RÜTHER, R.; SOLANO, J. Energy performance evaluation of a net plus-energy residential building with grid-connected photovoltaic system in Brazil. Energy and Buildings, v. 120, p. 19-29, 2016.

DEVORE J.L. Probabilidade e Estatística Para Engenharia e Ciências. 2 ed. São Paulo: Cengage Learning, p. 712, 2014.

EPE, Empresa De Pesquisa Energética. Análise da Inserção da Geração Solar na Matriz Elétrica Brasileira: Nota Técnica. Rio de Janeiro: EPE, p. 58, 2012.

EPE, Empresa de Pesquisa Energética. Balanço Energético Nacional 2017: Ano base 2016. Rio de Janeiro: EPE, p. 292, 2017a.

EPE, Empresa de Pesquisa Energética. Plano Decenal de Expansão de Energia 2026. Brasília: MME/EPE, p. 271, 2017b.

F. FREZATTI. Gestão da Viabilidade Econômico-Financeira dos Projetos de Investimento. São Paulo: Atlas, p. 144, 2008.

HOLDERMANN, C.; KISSEL, J.; BEIGEL, J. Distributed photovoltaic generation in Brazil: An economic viability analysis of small-scale photovoltaic systems in the residential and commercial sectors. Energy Policy, v. 67, p. 612-617, 2014.

IEC, International Electrotechnical Comission. System Performance Monitoring - Guidelines for Measurement, Data Exchange, and Analysis, IEC Standart 61724. Geneva: IEC 1998.

INMET. Instituto Nacional de Meteorologia. BDMEP - Dados Históricos. 2021. Disponível em https://portal.inmet.gov. $\mathrm{br} /$ servicos/bdmep-dados-hist $\% \mathrm{C} 3 \% \mathrm{~B} 3$ ricos, acesso em $10 / 02 / 2021$.

KANNAN, N.; VAKEESAN, D. Solar energy for future world: A review. Renewable and Sustainable Energy Reviews. v. 62, p. 1092-1105, 2016.

LIRA, M.A.T. Sistemas Híbridos Para o Fornecimento de Energia Elétrica na Comunidade Roça de Baixo, Paulistana (PI). Tese de Doutorado, Universidade Federal do Piauí, Teresina, 2015.

TAVARES, M. Estatística Aplicada à Administração. Brasília: Universidade Aberta do Brasil, p. 142, 2007.

MACHADO, A.F.; ALMEIDA, A.C.; ARAÚJO, A.C.; FERRARI, D.; LEMES, I.R.; FARIA, N.C.S.; LIMA, T.S.; FERNANDES, R.A. Aplicação de testes de normalidade em publicações nacionais: levantamento bibliográfico. Colloquium Vitae, v. 6, n. 1, p. 1-10, 2014.

MACÊDO, W.N.; ZILLES, R. Operational results of grid-connected photovoltaic system with different Inverter's Sizing Factors (ISF). Progress in Photovoltaics: Research and Applications. v. 15, p. 337-352, 2007.

MERMOUD, A.; WITTMER, B. PVsyst User's Manual: PVsyst 6. Satigny, Suíça, p. 102, 2014.

MONTEIRO JUNIOR, A.; SANTANA, K.G.S.; MACEDO, A.C.; NASCIMENTO, O.C.S.; SILVA, S.B. Análise de desempenho de sistemas fotovoltaicos com diferentes ângulos de inclinação e azimute localizados em Palmas-TO. In: 14 Conferência de Estudos em Engenharia Elétrica, Uberlândia, 2016. 
IFPI. Instituto Federal do Piauí. Usina Fotovoltáica do Campus Floriano Gera Economia de Mais de R\$ 200 mil. 2019. Disponível em https://www.ifpi.edu.br/floriano/noticias/ usina-fotovoltaica-do-campus-floriano-gera-economia-demais-de-r-200-mil, acesso em 10/07/2020.

MIOT, H.A. Avaliação da normalidade dos dados em estudos clínicos e experimentais. Jornal Vascular Brasileiro, v. 16 , n. 2, p. 88-91, 2017.

MORAIS, F.; MORAES, A.; BARBOSA, F. Technical-economic analysis of the first mini-generation photovoltaic system of Piauí, Brazil. IEEE Latin America Transactions, v. 17, n. 10, p. 1706-1714, 2019.

MOURA, A.D.; LUCAS, E.W.M.; RODRIGUES, J.E.; REZENDE, J.M. Nota Técnica $\mathrm{n}^{\circ}$ 001/2011/SEGER/ LAIME/ CSC/INMET. Rede de Estações Meteorológicas Automáticas do INMET. Brasília: INMET, 2011.

MUÑOZ, Y.; VARGAS, O.; PINILLA, G.; VÁSQUEZ, J. Sizing and study of the energy production of a grid-tied photovoltaic system using PVsyst software. Tecciencia, v. 12, n. 22, p. 27-32, 2017.

NAKABAYASHI, R.K. Microgeração Fotovoltaica no Brasil: Viabilidade Econômica. São Paulo: Instituto de Energia e Ambiente da Universidade de São Paulo, p. 58, 2015.

A BARBETTA, P.Estatística aplicada às Ciências Sociais. 9 ed. Florianópolis: Editora da Universidade Federal de Santa Catarina, p. 320, 2019

PARIDA, B.; INIYAN, S.; GOIC, R. A review of solar photovoltaic technologies. Renewable and Sustainable Energy Reviews, v. 15, p. 1625-163, 2011.

PEREIRA, E.B.; MARTINS, F.R.; GONÇALVES, A.R.; COSTA, R.S.; LIMA, F.J.L.; RÜTHER, R.; ABREU, S.L.; TIEPOLO, G.M.; PEREIRA, S.V.; SOUZA, J.G. Atlas Brasileiro de Energia Solar. 2.ed, São José dos Campos: INPE, p. 88, 2017.

PINHO, J.T.; GALDINO, M.A. Manual de Engenharia para Sistemas Fotovoltaicos. Rio de Janeiro: Centro de Pesquisas de Energia Elétrica / Centro de Referência para Energia Solar e Eólica Sérgio de Salvo Brito, p. 529, 2014.

PODEWILS, C. Differenze evidenti. Quel che i gestori degli impianti dovrebbero sapere sui programmi di simulazione. Photon. v. 5, p. 140-147, 2011.

SÁ, F.N.; SANTOS, F.F.P.; MORAES, A.M.; LOPES, O.S. Análise ambiental e econômica do primeiro ano de operação do sistema fotovoltaico de uma instituição federal de ensino no Brasil. Asociación Argentina de Energías Renovables y Medio Ambiente, v. 1, p. 1329-1336, 2017.

SAMPAIO, P.G.V.; GONZÁLEZ, M.O.A. Photovoltaic solar energy: Conceptual framework. Renewable and Sustainable Energy Reviews, v. 74, p. 590-601, 2017.

SILVA, L.R.J.R.; SHAYANI, R.A.; OLIVEIRA, M.A.G. Análise comparativa das fontes de energia solar fotovoltaica, hidrelétrica e termelétrica, com levantamento de custos ambientais, aplicada ao Distrito Federal. In: Anais VII Congresso Brasileiro de Energia Solar, Gramado, 2018.

SILVA, O.; ROCHA, F.; PINHEIRO, F. Viabilidade TécnicoEconômica da Eficiência Energética em Edificações. Curitiba: Appris, p. 173, 2019.

SWERA. Solar and Wind Energy Resource. Swera (beta release), 2017. Disponível em https://maps.nrel.gov/swera/ $\# /$ aL $=\mathrm{Hj} 0 \mathrm{X} 2 \mathrm{R} \% 255 \mathrm{Bv} \% 255 \mathrm{D} \% 3 \mathrm{Dt} \& \mathrm{bL}=$ groad $\&$ $\mathrm{cE}=0 \& 1 \mathrm{R}=0 \& \mathrm{mC}=-5.055463979126611 \% 2 \mathrm{C}$ $42.80140399932861 \& z \mathrm{~L}=16$, acesso em set/2017.

TONDOLO, V.A.G.; SCHNEIDER, L.C. A utilização de testes de hipótese paramétricos em pesquisas científicas. Global Manager, v. 6, n. 11, p. 147-167, 2006.

TORMAN, V.B.L.; COSTER, R.; RIBOLDI, J. Normalidade de variáveis: Métodos de verificação e comparação de alguns testes não-paramétricos por simulação. Clinical and Biomedical Research - Revista HCPA, v. 32, n. 2, p. 227234, 2012.

URBANETZ JUNIOR, J.; CASAGRANDE JUNIOR, E.F.; TIEPOLO, G.M. Análise do desempenho de dois anos de operação do sistema fotovoltaico conectado à rede elétrica do Escritório Verde da UTFPR. In: V Congresso Brasileiro de Energia Solar, 2014.

VALE, A.M.; FELIX, D.G.; FORTES, M.Z.; BORBA, B.S.M.C.; DIAS, B.H.; SANTELLI, B.S. Analysis of the economic viability of a photovoltaic generation project applied to the Brazilian housing program Minha Casa Minha Vida, Energy Policy, v. 108, p. 292-298, 2017.

VILlalva, M.G. Energia Solar Fotovoltaica: Conceitos e Aplicações. 2 ed. São Paulo: Érica, p. 224, 2015

License information: This is an open-access article distributed under the terms of the Creative Commons Attribution License (type CC-BY), which permits unrestricted use, distribution and reproduction in any medium, provided the original article is properly cited. 